\title{
A Rotational Period Study of a Large Sample of Pre-Main Sequence stars in NGC 2264
}

\author{
M. Lamm, C.A.L. Bailer-Jones, R. Mundt \\ MPI für Astronomie, Königstuhl 17, 69117 Heidelberg, Germany \\ W. Herbst \\ Van Vleck Obs., Wesleyan U., Middletown, CT 06459, USA
}

\begin{abstract}
We present the results of a photometric monitoring program of pre-main sequence (PMS) stars in the young (2-4 Myr) open cluster NGC 2264 $(\mathrm{d}=700 \mathrm{pc})$. We find that the rotation periods are mass dependent and show a bimodal distribution for higher mass stars with $M \gtrsim 0.3 \mathrm{M}_{\odot}$ and a unimodal distribution for lower mass stars with $M \lesssim 0.3 \mathrm{M}_{\odot}$.
\end{abstract}

\section{Introduction}

To investigate the role of circumstellar disks in the angular momentum evolution of pre-main sequence (PMS) stars it is necessary to obtain stellar rotation rates for objects at a large variety of masses and ages. Rotation periods can be determined from the periodic modulation of the stellar brightness caused by the large cool spots on the surface of PMS stars. Previous work in the Orion Nebula Cluster (ONC, e.g., Herbst et al. 2002) has shown that the distribution of rotational periods is mass dependent and bimodal for stars with $M>0.25 \mathrm{M}_{\odot}$. This was interpreted as an effect of a magnetic disk-star interaction (disk-locking).

\section{Observations \& Data Reduction}

We have carried out $I_{C}$-band observations of NGC 2264 on 44 nights from Dec. 2000 to Feb. 2001 using the Wide Field Imager (WFI) on the MPG/ESO 2.2m telescope on La Silla, Chile. Differential photometry relative to a set of nonvariable reference stars was performed for about 11000 stars in the field. All stars have been checked for both irregular and periodic variability using a $\chi^{2}$ test and the periodogram technique described by Scargle (1982) respectively. Absolute aperture photometry was performed for $V, R_{C}, I_{C}$ and $H \alpha$.

\section{Results}

Probable cluster members among the periodic and irregular variable stars in the field were selected according to their locations in the $\left(R_{C}-I_{C}\right), I_{C}$ colormagnitude and $\left(R_{C}-I_{C}\right),\left(R_{C}-H \alpha\right)$ color-color diagrams. The final sample of probable PMS stars contains 404 periodic and 228 irregular variable stars. 

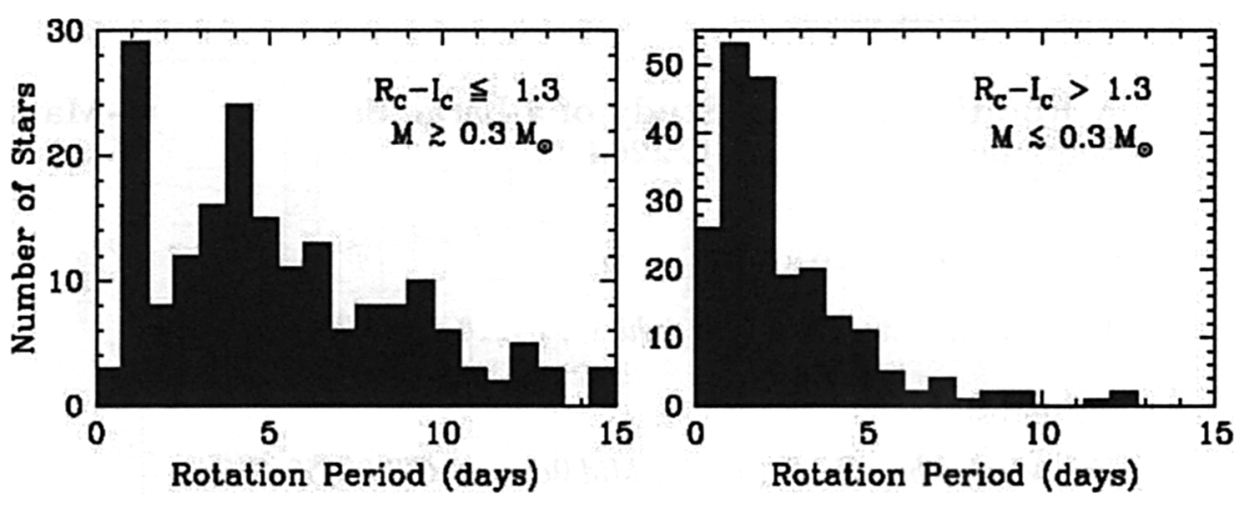

Figure 1. The period distribution of periodic variable stars in NGC 2264 for 187 higher mass stars (left panel) and 217 lower mass stars (right panel).

In order to compare the period distribution we found for NGC 2264 (age: 2-4 Myr) with that was found for the ONC (age: $1 \mathrm{Myr}$ ) by Herbst et al. (2001 $\& 2002$ ) we have divided the periodic stars into two sub samples according to the color of the stars as indicated in Fig. 1. This corresponds roughly to a division into two mass regimes: $1.5 \mathrm{M}_{\odot} \lesssim M \lesssim 0.3 \mathrm{M}_{\odot}$ and $M \lesssim 0.3 \mathrm{M}_{\odot}$. In Fig. 1 we show the period distribution for both samples. It is clear that the period distribution is color dependent. For the higher mass stars it is bimodal with peaks at about 1 and 4 days and for lower mass stars the distribution is unimodal. Compared to the younger ONC we find that in NGC 2264 the peaks in both the bimodal distribution of the higher mass stars and in the unimodal distribution of the lower mass stars are at about half of the values measured for the ONC. If one assumes equal initial conditions in both clusters and that the stars in NGC 2264 are no longer magnetically locked to their disks (i.e. their angular momentum is constant and $P \propto R^{2} \propto t^{-2 / 3}$ ) their higher rotational speed compared to the ONC stars can be interpreted simply as a result of their larger age and correspondingly smaller stellar radius. However, if a significant fraction of the stars is still locked to their disks the shorter periods (especially of the long periodic stars with $P>2.5 \mathrm{~d}$ ) may also be caused by changes in the locking period, dependent on the stellar magnetic field and accretion rate. That indeed some stars are still disk locked is supported by our $H \alpha$ data. We found that for both samples in Fig. 1 the fraction of stars with strong $H \alpha$ emission is larger by a factor of 2.5 among the slow rotators.

\section{References}

Herbst, W., Bailer-Jones, C. A. L., Mundt, R. 2001, ApJ 554, L197

Herbst, W., Bailer-Jones, C. A. L., Mundt, R., et al. 2002, 396, 513

Scargle, J.D. 1982, ApJ 263, 835 